\title{
Dynamic Activity Management in Many-to-one Sensor Networks
}

\author{
Zhifeng $\mathrm{Hu}$ and Junshan Zhang \\ Dept. of Electrical Engineering \\ Arizona State University \\ Tempe, AZ 85287 \\ \{zhifeng.hu, junshan.zhang\}@asu.edu
}

\author{
Lang Tong \\ School of Electrical and Computer Engineering \\ Cornell University \\ Ithaca, NY 14853 \\ ltong@ece.cornell.edu
}

\section{ABSTRACT}

We consider a many-to-one sensor network in which a large number of sensors are deployed to monitor an environment. We study sensor activity management therein to maximize the network lifetime while meeting the network resolution requirement. Specifically, in each transmission round the sink estimates the number of active sensors and broadcasts control information to the sensors for activity management. We first consider the case with accurate estimation, and devise a sensor activity control scheme under which the number of active sensors would converge to the minimum one that can meet the requirement. Next, we study the case when the estimation is inaccurate, and propose a stochastic approximation method to minimize the average number of active sensors.

\section{INTRODUCTION}

In recent years, the rapid advances in microelectro-mechanical systems and wireless technologies have enabled the integration of monitoring and wireless communication capabilities into sensor devices. When a large number of cheap sensors are distributed over an area to monitor a physical environment or to detect chemical and biological warfare agents, the sensors can form a many-to-one sensor network to report the data to a central unit. Such networks can provide valuable information regarding the physical phenomena of interest and thereby enable us to detect and control them. Potential applications of these networks include environmental monitoring, home security, battlefield surveillance and reconnaissance, etc.

The focus of this study is on the quality of service(QoS) control in many-to-one sensor networks. This is a relatively lessunderstood area, partially due to the difficulty in defining and supporting QoS for a variety of sensor network models. In this paper, the QoS, to be defined more precisely, can be viewed as the network resolution and is directly related to the number of sensors actively working in the network. Needless to say, the highest QoS can be achieved when all the sensors are used to monitor the environment, at the cost of a shortened network lifetime, due to the energy constraints of the sensors. Then, it is desirable to maintain a minimum number of active sensors while still meeting the QoS requirements. To this end, we devise a mechanism to dynamically control the number of active sensors.

\footnotetext{
${ }^{0}$ This research is supported in part by Office of Naval Research through the grant N00014-05-1-0636 and National Science Foundation through the CAREER award ANI-0238550.
}

The main objective is to minimize the energy consumption in the network while satisfying the QoS requirement, thus ensuring the best possible energy efficiency.

Specifically, we consider a model where there are a central sink and $N$ sensors distributed over the surveillance area. Each sensor has two states, namely ON and OFF. Once a sensor is switched to $\mathrm{ON}$, it monitors the environment, generates data packets and transmits them to the sink. A sensor can switch to OFF to save energy when there are more than enough sensors working in the network. The sink collects packets from the active sensors and reconstructs the phenomenon being monitored. We assume that there is a separate broadcast channel from the sink to the sensors, and that each sensor listens to this channel and decides its activity in the next round using the feedback information.

For convenience, let $n_{t}$ denote the number of active sensors in round $t$, and $\hat{n}_{t}$ is an estimator of $n_{t}$. The QoS here is defined as the distortion between the original physical phenomenon and its reconstructed version at the sink. We consider a homogeneous network, for which we assume that there is a one-one mapping between $n_{t}$ and the distortion, denoted as $D\left(n_{t}\right)$. Throughout, we assume that the sink knows the exact form of $D\left(n_{t}\right)$ and adjusts the number of active sensors accordingly. The QoS requirement is given as

$$
D\left(n_{t}\right) \leq D_{o}
$$

The basic idea of dynamic sensor activity management can be outlined as follows. Let $n_{o p}$ denote the operational number of active sensors in the network. That is, $n_{o p}$ is the targeted number of active sensors in the network. At the beginning of each round, the number of active sensors in the network is estimated at the sink. If $\hat{n}_{t} \leq n_{o p}$, the sink computes a probability $p_{1}$ as $p_{1}=\frac{n_{o p}-\hat{n}_{t}}{N-\hat{n}_{t}}$ and broadcasts it. Each dormant sensor would then switch to ON with probability $p_{1}$ in the next round; in contrast, if $\hat{n}_{t}>n_{o p}$, the sink computes $p_{2}$ as $p_{2}=\frac{\hat{n}_{t}-n_{o p}}{\hat{n}_{t}}$, and each active sensor would switch to OFF accordingly. First, we assume $\hat{n}_{t}$ is accurate for simplicity and study sensor activity management therein. Observing that in practical scenarios $\hat{n}_{t}$ may not be accurate, we also study sensor activity management when $\hat{n}_{t}$ is inaccurate. Our main contributions can be outlined as follows:

1) The case with accurate $\hat{n}_{t}$. In this case, when the above activity management scheme with a given $n_{o p}$ is applied, $\left\{n_{t}\right\}$ evolves as a Markov chain. We show that $n_{t}$ converges to $n_{o p}$ with probability one in steady state. As a result, if $n_{o p}$ is set to be $n_{a}^{*}$, where $n_{a}^{*}$ is the minimum $n_{t}$ 
satisfying $D\left(n_{t}\right) \leq D_{o}$, the system would maintain the minimum number of active sensors while satisfying the QoS requirement.

2) The case with inaccurate $\hat{n}_{t}$. There has been a great deal of interest in estimating the number of active sensors in sensor networks [2], [8]. In some scenarios, it may be difficult to obtain perfect estimation of $n_{t}$. If for a given $n_{o p}$ the control scheme is applied to the system when $\hat{n}_{t}$ is inaccurate, $n_{t}$ would evolve as a Markov chain, and would be a random variable even in steady states. To minimize the network energy consumption while satisfying the QoS constraint, we devise an algorithm based on a stochastic approximation approach to adaptively adjust $n_{o p}$. We show that under this new sensor activity control scheme, the average number of active sensors is minimized while $D \leq D_{o}$ is satisfied.

The paper is organized as follows: in Section II, we review some related work on sensor networks. In Section III, we consider the case where $\hat{n}_{t}$ is accurate, and propose a scheme for sensor activity management accordingly. We prove that using this scheme $\left\{n_{t}\right\}$ would converge to $n_{a}^{*}$ with probability one. In Section IV, we study the case where $\hat{n}_{t}$ is inaccurate. In this case, we use a stochastic approximation method to adapt $n_{o p}$ so that the average number of active sensors is minimized in steady state while the QoS requirement is still satisfied.

\section{RELATED WORK}

Due to their wide range of potential applications, many-to-one sensor networks have recently received much attention from the research community. In this section, we give a brief review of related work in this field.

The capacity and energy efficiency of many-to-one sensor networks have been two heavily studied fields due to their importance to the performance of such networks. In [9], the authors investigate the capability of a large-scale sensor network to measure and transport independent snap-shots of a two dimensional field to the central sink. An information theoretic approach is taken in [1] to completely characterize the source/channel capacity of the reachback channel, where a common receiver collects from multiple sensors local measurements of a random field to reproduce it. In the field of energy efficiency, the work in [3] analyzes the energy consumption in a many-to-one sensor network. Both flat and clustering architectures are considered. The authors of [4] study how to maximize the lifetime of a sensor network for a given amount of energy, or equivalently, how to retrieve the same data using the least amount of energy.

The idea of using a feedback control scheme to control the performance of a network is not new. In [12], the authors study a price-based rate control mechanism for random access networks which is analyzed using the slotted Aloha model with an infinite set of nodes. The mechanism uses channel feedback information to control the aggregate packet arrival rate. The parameters of the rate control scheme can be chosen a priori to stabilize the system at a desired operating point. Similarly, our scheme uses channel feedback information to control the number of active sensors, which decides the amount of incoming data. Also, in our scheme, $n_{o p}$ is adjustable so that the number of active sensors in steady state is controlled.
Most relevant to our work is perhaps [7] in which sensor network resolution is investigated. Assuming the quality of service(QoS) is defined as the optimum number of sensors sending information to the sink, [7] presents a sensor activity control scheme via using the Gur Game. In their approach, however, the sink needs to receive successful transmissions from all the active sensors in order to know the exact number of active sensors in each round, and each sensor is required to maintain an automaton. As a result, the complexity is relatively high.

\section{Sensor Activity Management for the Case With ACCURATE $\hat{n}_{t}$}

\section{A. Sensor Activity Control}

For any given $n_{o p}$, after $n_{t}$ is estimated at the sink at the beginning of each round, the probabilities $p_{1}$ and $p_{2}$ are computed as

$$
\begin{aligned}
& p_{1}=\frac{n_{o p}-\hat{n}_{t}}{N-\hat{n}_{t}} \mathcal{I}\left(\hat{n}_{t} \leq n_{o p}\right) \\
& p_{2}=\frac{\hat{n}_{t}-n_{o p}}{\hat{n}_{t}} \mathcal{I}\left(\hat{n}_{t}>n_{o p}\right),
\end{aligned}
$$

and are broadcast to all the sensors. Then the sensors manage their activities probabilistically as mentioned before. The above feedback control scheme is locally optimal in each round in the sense that in computing $p_{1}$ and $p_{2}$, it maximizes the probability of transitting to the desired state $n_{t}=n_{o p}$ in the next round.

Clearly, the number of active sensors in the network evolves as

$$
n_{t+1}=n_{t}+X_{t}^{a}-X_{t}^{b}
$$

where $X_{t}^{a}$ and $X_{t}^{b}$ are Binomial random variables, and

$$
\begin{aligned}
X_{t}^{a} & \sim \operatorname{Binomial}\left(N-n_{t}, p_{1}\right) \\
X_{t}^{b} & \sim \operatorname{Binomial}\left(n_{t}, p_{2}\right) .
\end{aligned}
$$

Note that $\left\{n_{t}\right\}$ evolves as a Markov chain with states $1,2, \ldots N$. Since $\hat{n}_{t}$ is exactly $n_{t}$ in the accurate estimation case, it is clear that the transition probability from the state $n_{t}=n_{o p}$ to any other state is zero, i.e., $P\left(n_{t+1} \neq n_{o p} \mid n_{t}=n_{o p}\right)=0$. That is to say, $n_{t}=n_{o p}$ is the absorbing state of the Markov chain, and all the other states are transient states. The stationary distribution is then

$$
P\left(n_{t}=n\right)= \begin{cases}1 & \text { if } n=n_{o p} \\ 0 & \text { if } n \neq n_{o p}\end{cases}
$$

It is clear that $\left\{n_{t}\right\}$ converges to $n_{o p}$ with probability one. Therefore, if we choose $n_{o p}=n_{a}^{*}$, where

$$
n_{a}^{*}=\min \left\{n_{t}: D\left(n_{t}\right) \leq D_{o}\right\},
$$

the system would have the minimum number of active sensors while satisfying the distortion constraint when the feedback control scheme is applied to it.

Next, we characterize the absorption time of the Markov chain, defined as the expected time it takes the system to arrive at the absorbing state. To this end, we write the transition matrix of the Markov chain as:

$$
p=\left(\begin{array}{l|l}
S & T \\
\hline 0 & 1
\end{array}\right),
$$

where the $(i j)$-th element $p_{i j}=P\left(n_{t+1}=j \mid n_{t}=i\right)$ and the index of the states are arranged such that $n_{t}=n_{o p}$ is the last one. Note 


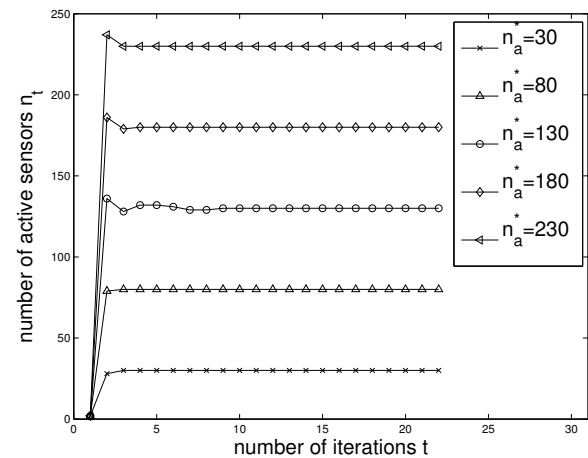

Fig. 1. Convergence performance for different $n_{a}^{*}$

that $S$ is a square $(N-1) *(N-1)$ matrix denoting the transition probabilities for movement among the non-absorbing states, and $T$ is a column vector denoting the transition probabilities for movement from the non-absorbing states to the absorbing state, $n_{t}=n_{a}^{*}$. Define $Q \triangleq(I-S)^{-1}$, where $q_{i j}$ stands for the number of times the system is expected to visit state $j$ before absorption [6]. As a result, the absorption time starting with state $i, t_{i}$, is the sum of all the entries in the $i$-th row of $Q$.

\section{B. Numerical Examples}

In the following, we illustrate by numerical examples the convergence of $\left\{n_{t}\right\}$ under the above feedback control scheme. In the simulation, we set $N=300$, and let $n_{a}^{*}$ take on different values. As shown in Fig. 1, it takes only a few iterations for $\left\{n_{t}\right\}$ to converge to $n_{a}^{*}$ for all cases.

\section{Sensor Activity Management for the Case With INACCURATE $\hat{n}_{t}$}

\section{A. Problem Formulation}

As shown in the previous section, when the estimation is accurate, the number of active sensors in the system would converge to $n_{a}^{*}$. However, practical algorithms may not achieve perfect estimation of $n_{t}$ in some scenarios. In this section, we study how to carry out dynamic activity management when $\hat{n}_{t}$ is inaccurate.

For a given $n_{o p}$, if the above scheme is applied using an inaccurate estimator $\hat{n}_{t},\left\{n_{t}\right\}$ evolves as a Markov chain. Furthermore, even if $n_{t}$ equals $n_{o p}$ at certain time instant, it can still transit to other states due to the inaccuracy in $\hat{n}_{t}$. In a nutshell, $\left\{n_{t}\right\}$ evolves as an irreducible Markov chain and would no longer converge to $n_{o p}$ with probability one. The average distortion in steady state, denoted as $T\left(n_{o p}\right)$, now is

$$
T\left(n_{o p}\right)=\sum_{n=0}^{N} D(n) P\left(n_{t}=n \mid n_{o p}\right),
$$

where we have abused the notation by using $P\left(n_{t} \mid n_{o p}\right)$ to denote the stationary distribution of $\left\{n_{t}\right\}$ when the control scheme is applied to the system with a given $n_{o p}$.

As a result, if we still let $n_{o p}$ take on the value of $n_{a}^{*}$, the QoS requirement $T\left(n_{o p}\right) \leq D_{o}$ may no longer be satisfied. Therefore, it is critical to determine a new $n_{o p}$ to ensure that $T\left(n_{o p}\right) \leq D_{o}$ while minimizing the average number of active sensors, which is given by

$$
\boldsymbol{E}\left[n_{t}\right]=\sum_{n=0}^{N} n P\left(n_{t}=n \mid n_{o p}\right) .
$$

In a nutshell, the problem boils down to the following optimization problem:

$$
\begin{aligned}
P 1: & \min _{n_{o p}} \boldsymbol{E}\left[n_{t}\right] \\
\text { s. t. } & T\left(n_{o p}\right) \leq D_{o} .
\end{aligned}
$$

\section{B. A Stochastic Approximation Approach}

Given $n_{o p}$, the distortion function $T\left(n_{o p}\right)$ depends on the stationary distribution of $\left\{n_{t}\right\}$ and hence the state-dependent transition matrix, which are difficult to characterize in general. That is to say, the exact form of $T\left(n_{o p}\right)$ is not available and P1 cannot be solved directly.

For a given $n_{t}$, let $D\left(\hat{n}_{t} \mid n_{t}\right)$ denote the distortion computed from $\hat{n}_{t}$, i.e., $D\left(\hat{n}_{t} \mid n_{t}\right) \triangleq D\left(\hat{n}_{t}\right)$, where $\hat{n}_{t} \sim P\left(\hat{n}_{t} \mid n_{t}\right)$. We need the following assumption:

Condition 1: We assume that $\boldsymbol{E}_{\hat{n}_{t}}\left[D\left(\hat{n}_{t} \mid n_{t}\right)\right] \geq D\left(n_{t}\right)$.

Intuitively speaking, Condition 1 requires that for a given $n_{t}$, the expectation of the distortion computed from the estimator be greater than or equal to the actual distortion $D\left(n_{t}\right)$. Note that Condition 1 holds in many cases of practical interest. For example, it holds when $D\left(n_{t}\right)$ is a convex function and the estimator $\hat{n}_{t}$ is unbiased. In [5], it is shown that in a Gaussian sensor network, the distortion as a function of the number of active sensors is in the form of

$$
D_{1}\left(n_{t}\right)=\frac{1}{a+b n_{t}} .
$$

It is easy to verify that $D_{1}\left(n_{t}\right)$ is convex. Therefore, when the estimator is unbiased, i.e., $\boldsymbol{E}_{\hat{n}_{t}}\left[\hat{n}_{t} \mid n_{t}\right]=n_{t}$, we have that

$$
\boldsymbol{E}_{\hat{n}_{t}}\left[D_{1}\left(\hat{n}_{t} \mid n_{t}\right)\right] \geq D_{1}\left(\boldsymbol{E}_{\hat{n}_{t}}\left[\hat{n}_{t} \mid n_{t}\right]\right)=D_{1}\left(n_{t}\right) .
$$

For convenience, let $D\left(\hat{n}_{t} \mid n_{o p}\right)$ denote the distortion for a given $n_{o p}$, i.e., $D\left(\hat{n}_{t} \mid n_{o p}\right) \triangleq D\left(\hat{n}_{t}\right)$, where $\hat{n}_{t} \sim \sum_{n_{t}=0}^{N} P\left(\hat{n}_{t} \mid\right.$ $\left.n_{t}\right) P\left(n_{t} \mid n_{o p}\right)$. The expectation of $D\left(\hat{n}_{t} \mid n_{o p}\right)$ with respect to $\hat{n}_{t}$ would be

$$
\begin{aligned}
& \boldsymbol{E}_{\hat{n}_{t}}\left[D\left(\hat{n}_{t} \mid n_{o p}\right)\right] \\
= & \sum_{j=0}^{N} \sum_{i=0}^{N} D(i) P\left(\hat{n}_{t}=i \mid n_{t}=j\right) P\left(n_{t}=j \mid n_{o p}\right) .
\end{aligned}
$$

Denote it as $\bar{D}\left(n_{o p}\right)$. Under Condition 1, it follows that

$$
\begin{aligned}
& \bar{D}\left(n_{o p}\right) \\
= & \sum_{j=0}^{N} \sum_{i=0}^{N} D(i) P\left(\hat{n}_{t}=i \mid n_{t}=j\right) P\left(n_{t}=j \mid n_{o p}\right) \\
= & \sum_{j=0}^{N} \boldsymbol{E}_{\hat{n}_{t}}\left[D\left(\hat{n}_{t} \mid n_{t}=j\right)\right] P\left(n_{t}=j \mid n_{o p}\right) \\
\geq & \sum_{j=0}^{N} D(j) P\left(n_{t}=j \mid n_{o p}\right) \\
= & T\left(n_{o p}\right) .
\end{aligned}
$$


That is to say, $\bar{D}\left(n_{o p}\right) \geq T\left(n_{o p}\right)$ under Condition 1. Therefore, a sufficient condition to meet the constraint in P1 is that $\bar{D}\left(n_{o p}\right) \leq$ $D_{o}$. Therefore, a "suboptimal" solution to P1 can be obtained by solving the following problem:

$$
\begin{aligned}
P 2: & \min _{n_{o p}} \boldsymbol{E}\left[n_{t}\right] \\
\text { s. t. } & \bar{D}\left(n_{o p}\right) \leq D_{o} .
\end{aligned}
$$

For convenience, let $n_{c}^{*}$ denote the solution to P2. Since $\bar{D}\left(n_{o p}\right)$ is monotonic decreasing and $\boldsymbol{E}\left[n_{t}\right]$ in steady state is a monotonic increasing function of $n_{o p}, n_{c}^{*}$ would be the root of the equation $\bar{D}\left(n_{o p}\right)=D_{o}$. Observe that $D\left(\hat{n}_{t} \mid n_{o p}\right)$, a random variable with expectation equal to $\bar{D}\left(n_{o p}\right)$, can always be directly computed from $\hat{n}_{t}$. With $D\left(\hat{n}_{t} \mid n_{o p}\right)$ available, a standard stochastic approximation approach can be used to solve P2. Accordingly, we develop a stochastic approximation algorithm (see in Algorithm I) in what follows.

\section{Algorithm I}

1) Initial phase: let $k=1$ and $i=0$; choose the value of $n_{1}$ assuming accurate estimation case;

2) If $n_{k}>N$, let $n_{k+1}=N$ and go to Step 5. If $n_{k}<0$, let $n_{k+1}=0$ and go to Step 5. Let $i=i+1$. Estimate the number of active sensors in the current round as $\hat{n}_{k}^{i}$;

3) If $i=i_{o}$, go to Step 4 . If $i<i_{o}$, the sink computes $p_{1}$ or $p_{2}$ according to (2) with $n_{o p}=n_{k}$ and $\hat{n}_{t}=\hat{n}_{k}^{i}$ and broadcasts it to the sensors. The sensors switch to ON/OFF accordingly. Go back to Step 2;

4) Compute the distortion from the estimator $\hat{n}_{k}^{i_{o}}$ as $D_{k}=$ $D\left(\hat{n}_{k}^{i_{o}}\right)$. Update $n_{k}$ as

$$
n_{k+1}=n_{k}+a_{k}\left(D_{k}-D_{o}\right),
$$

where $\left\{a_{k}\right\}$ is a pre-specified positive decreasing sequence;

5) Let $k=k+1$ and $i=0$. Go back to Step 2 .

The basic idea of Algorithm I is as follows: start with $n_{1}$ set to $n_{a}^{*}$. For each $k$, apply the control scheme defined in (2) with $n_{o p}=n_{k}$ to the system for $i_{o}-1$ consecutive rounds. At the end of the last round, use the estimator $\hat{n}_{k}^{i_{o}}$ to compute the distortion $D_{k}$. Compare $D_{k}$ with $D_{o}$ and use the difference to update $n_{k}$. The objective of the algorithm is to obtain a sequence $\left\{n_{k}\right\}$ that will converge to $n_{c}^{*}$ and therefore solve P2.

Note that for each $k$, if the control scheme with $n_{o p}=n_{k}$ is applied to the system for $i_{o}+l-2$ consecutive rounds and the average value of the estimators obtained in the last $l$ rounds is used to compute $D_{k}$ instead of $\hat{n}_{k}^{i_{o}}$, i.e., $D_{k}=D\left(\frac{1}{l} \sum_{j=0}^{l-1} \hat{n}_{i_{o}+j}^{k}\right)$, the convergence rate of the sequence $\left\{n_{k}\right\}$ might change. Also, the choice of $i_{o}$ can affect the convergence rate. In the next section, numerical results will show that generally $\left\{n_{k}\right\}$ converges faster when $i_{o}=2$ and $l=1$.

Next, we prove that the sequence $\left\{n_{k}\right\}$ as obtained in Algorithm I converges to $n_{c}^{*}$ in probability. First, we show some properties of the sequence $\left\{D_{k}\right\}$ that will be used to prove the convergence of the sequence $\left\{n_{k}\right\}$. Given $n_{o p}=n_{k}$, assuming that the system has arrived at steady state in the $i_{o}$ rounds before $D_{k}$ is computed, we have that $D_{k}=D\left(\hat{n}_{t} \mid n_{o p}=n_{k}\right)$ and $\boldsymbol{E}_{\hat{n}_{k}^{i_{o}}}\left[D_{k}\right]=\bar{D}\left(n_{k}\right)$.

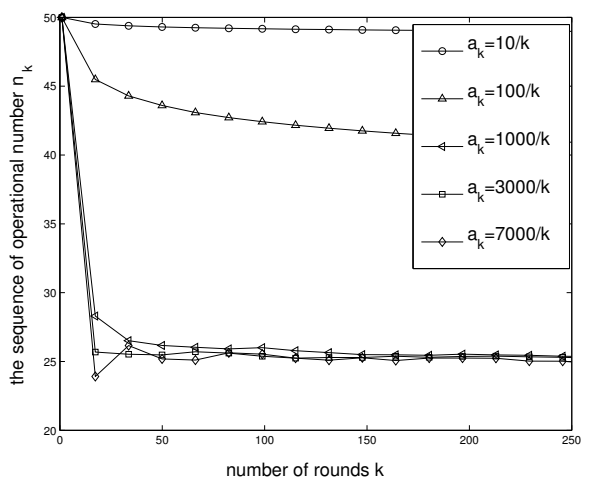

Fig. 2. Convergence performance for different $\left\{a_{k}\right\}$

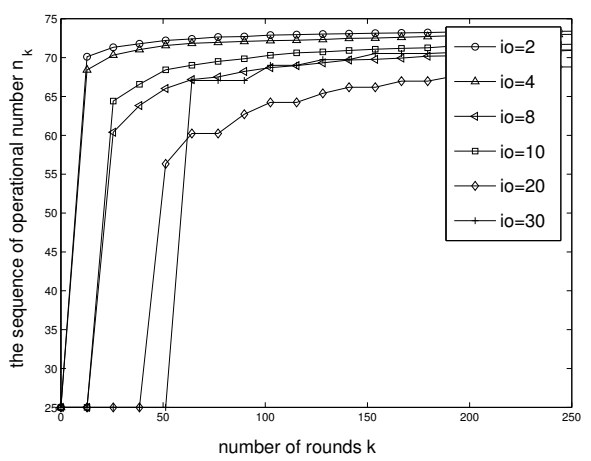

Fig. 3. Convergence performance for different $i_{o}$

For convenience, define

$$
\begin{aligned}
& b_{k} \triangleq-\frac{\bar{D}\left(n_{k}\right)-D_{o}}{n_{k}-n_{c}^{*}} \\
& e_{k} \triangleq D_{k}-\bar{D}\left(n_{k}\right),
\end{aligned}
$$

we have the following lemma:

Lemma 4.1: There exist constants $c_{0}, c_{1}, c_{2}>0$ such that

1) $c_{o} \leq b_{k} \leq c_{1}, \forall k \in \mathbb{N}$

2) $\boldsymbol{E}\left[e_{k}\right]=0,\left|e_{k}\right| \leq c_{2}, \forall k \in \mathbb{N}$.

Now we prove that the sequence $\left\{n_{k}\right\}$ obtained from (7) converges to $n_{c}^{*}$ in probability using Lemma 4.1. This is shown by the following theorem:

Theorem 4.1: There exists a sequence $\left\{a_{k}\right\}$ for Algorithm I such that when the sequence $\left\{a_{k}\right\}$ is used, $\left\{n_{k}\right\}$ would converge to $n_{c}^{*}$ in probability, i.e., there exists a constant $c_{3}$ such that

$$
\begin{aligned}
\lim _{k \rightarrow \infty} \boldsymbol{E}\left[n_{k}\right] & =n_{c}^{*} \\
\lim _{k \rightarrow \infty} k * \operatorname{var}\left(n_{k}-n_{c}^{*}\right) & \leq c_{3} \\
\lim _{k \rightarrow \infty} P\left(\left|n_{k}-n_{c}^{*}\right|>\epsilon\right) & =0, \forall \epsilon>0 .
\end{aligned}
$$

The proof of Theorem 4.1 has been relegated to Appendix I.

\section{Numerical Results}

In this section, we illustrate by numerical examples the convergence of Algorithm I, and study the effects of different $i_{o}$ and $l$ 


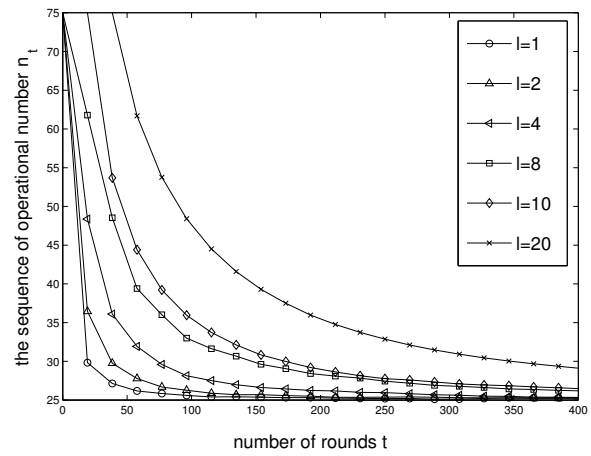

Fig. 4. Convergence performance for different $l$

on the rate of convergence. Throughout the simulations, we set $N=100, D\left(n_{t}\right)=\frac{1}{n_{t}}$ and $\hat{n}_{t}=n_{t}+z$, where $z \sim N(0,1)$.

To verify the convergence of the sequence $\left\{n_{k}\right\}$, we fix $i_{o}=$ 2 and $l=1$, and choose different pairs of $D_{o}$ and $n_{1}$. For each pair of $D_{o}$ and $n_{1}$, we let $a_{k}$ take on different values in $\left\{\frac{10}{k}, \frac{100}{k}, \frac{1000}{k}, \frac{3000}{k}, \frac{7000}{k}\right\}$, and investigate the convergence behavior of $\left\{n_{k}\right\}$. As shown in Fig. 2, $\left\{n_{k}\right\}$ converges when $a_{k}$ takes on the values in $\left\{\frac{1000}{k}, \frac{3000}{k}, \frac{7000}{k}\right\}$, but may not converge when $a_{k}$ equals $\frac{10}{k}$ or $\frac{100}{k}$. This corroborates the fact that it is necessary to have $a_{k}>\frac{1}{c_{0} k}$, as pointed out in the proof of Theorem 4.1.

Next, we investigate the impact of $i_{o}$ and $l$ on the convergence rate of $\left\{n_{k}\right\}$. Intuitively, increasing $i_{o}$ and $l$ would make the time the system stays at $n_{k}$ longer, but it is also likely to decrease the number of rounds necessary for convergence. To get a more concrete sense, we investigate the convergence rate under several numeric values of $i_{o}$ and $l$. We set $l=1$ and let $i_{o}$ take on different values in $\{2,4,8,10,20,30\}$. To compare the convergence rates on the same time scale, $\left(i_{o}+l\right) k$ is used for the horizontal axis instead of $k$. It can be seen from Fig. 3 that the fastest convergence rate is achieved when $i_{o}$ equals 2 . This indicates that the increment in the time length of every round outweighs the decrement in the number of rounds necessary for convergence when $i_{o}$ is increased. Finally, in Fig. 4, we set $i_{o}=2$ and investigate the impact of $l$ on the convergence rate. The value of $l$ is chosen from $\{1,2,4,8,10,20\}$. It can be seen that the fastest convergence rate occurs when $l=1$.

\section{Conclusion}

In this paper, we studied dynamic sensor activity management in many-to-one sensor networks, aiming to maximize the network lifetime while meeting the QoS requirement. We assume that in each transmission round the sink estimates the number of active sensors and broadcasts control information to the sensors for activity management. We consider the following two cases: 1) the estimator, $\hat{n}_{t}$, is accurate, and 2) $\hat{n}_{t}$ is inaccurate. When $\hat{n}_{t}$ is accurate, we devised a sensor activity control scheme under which the number of active sensors would converge to the minimum one that can meet the QoS requirement. When $\hat{n}_{t}$ is inaccurate, we proposed a stochastic approximation method to dynamically update the control scheme so that the average number of active sensors is minimized while the QoS requirement is satisfied. This scheme is applicable to many practical scenarios with inaccurate estimations

A concern that may arise is that some active nodes may remain active until they die after reaching steady state. To address this issue, it is plausible to turn off active sensors when their energy reserve fall below a certain threshold, and these sensors may remain off for a certain period of time. As a result, the total number of sensors in the network varies. Furthermore, in many practical scenarios, sensor deaths and replenishment can result in an unknown total number of sensors. Therefore, it is of great interest to generalize the study to scenarios where the total number of sensors in the network is unknown to the sink, and we are currently investigating this problem.

\section{APPENDIX I \\ ProOF OF THEOREM 4.1}

Let $a_{k}=\frac{c_{4}}{k}$, where $c_{4}>\frac{1}{c_{0}}$. Then from Lemma 4.1, we have that

$$
\begin{aligned}
& a_{k} b_{k}>1 / k, \forall k \in \mathbb{N} \\
& a_{k} b_{k}<1, \forall k>k_{o},
\end{aligned}
$$

where $k_{o}$ is the smallest integer greater than $c_{4} c_{1}$.

Plugging $b_{k}$ and $e_{k}$ into (7) yields that

$$
n_{k+1}-n_{c}^{*}=\left(n_{k}-n_{c}^{*}\right)\left(1-a_{k} b_{k}\right)+e_{k} a_{k} .
$$

Letting $m_{k}=\sqrt{k}\left(n_{k}-n_{c}^{*}\right)$, we have that

$$
\begin{aligned}
m_{k}= & \sqrt{k} m_{1} \prod_{j=1}^{k-1}\left(1-a_{j} b_{j}\right)+ \\
& \sqrt{k} \sum_{i=1}^{k-1}\left(e_{i} a_{i} \prod_{j=i+1}^{k-1}\left(1-a_{j} b_{j}\right)\right) .
\end{aligned}
$$

To determine the mean value and the variance of $m_{k}$, let $u_{k}$ and $v_{k}$ denote the first term and the second term on the right hand side of the equation, respectively. Since each term in $v_{k}$ contains $e_{i}$, which is independent of $a_{j}$ and $b_{j}$ for any $j, v_{k}$ is independent of $u_{k}$. It follows that

$$
\begin{aligned}
\boldsymbol{E}\left[m_{k}\right] & =\boldsymbol{E}\left[u_{k}\right]+\boldsymbol{E}\left[v_{k}\right] \\
\operatorname{var}\left(m_{k}\right) & =\operatorname{var}\left(u_{k}\right)+\operatorname{var}\left(v_{k}\right) .
\end{aligned}
$$

Next we characterize the mean value and the variance of $u_{k}$.

$$
\begin{aligned}
& \left|u_{k}\right| \\
= & \sqrt{k}\left|m_{1}\right| \prod_{j=1}^{k-1}\left|1-a_{j} b_{j}\right| \\
= & \sqrt{k}\left|m_{1}\right| \prod_{j=1}^{k_{o}}\left|1-a_{j} b_{j}\right| \prod_{j=k_{o}+1}^{k-1}\left|1-a_{j} b_{j}\right| \\
\leq & \sqrt{k}\left|m_{1}\right| \prod_{j=1}^{k_{o}}\left(1+\frac{c_{1} c_{4}}{j}\right) \prod_{j=k_{o}+1}^{k-1}\left(1-\frac{1}{j}\right) \\
= & \sqrt{k}\left|m_{1}\right| \frac{k_{o}}{k-1} \prod_{j=1}^{k_{o}}\left(1+\frac{c_{1} c_{4}}{j}\right) .
\end{aligned}
$$

Therefore $\lim _{k \rightarrow \infty}\left|u_{k}\right|=0$ and $\lim _{k \rightarrow \infty} \boldsymbol{E}\left[u_{k}\right]=0$. 
Observe that

$$
\begin{aligned}
& \operatorname{var}\left(u_{k}\right) \\
= & \boldsymbol{E}\left[u_{k}^{2}\right]-\boldsymbol{E}^{2}\left[u_{k}\right] \\
= & \boldsymbol{E}\left[\left(\sqrt{k} m_{1} \prod_{j=1}^{k-1}\left(1-a_{j} b_{j}\right)\right)^{2}\right] \\
= & \boldsymbol{E}\left[\left(\sqrt{k} m_{1}\right)^{2} \prod_{j=1}^{k_{o}}\left(1-a_{j} b_{j}\right)^{2} \prod_{j=k_{o}+1}^{k-1}\left(1-a_{j} b_{j}\right)^{2}\right] \\
\leq & \left(\sqrt{k} m_{1}\right)^{2} \prod_{j=1}^{k_{o}}\left(1+\frac{c_{1} c_{4}}{j}\right)^{2} \prod_{j=k_{o}+1}^{k-1}\left(1-\frac{1}{j}\right)^{2} \\
= & \left(\sqrt{k} m_{1}\right)^{2} \prod_{j=1}^{k_{o}}\left(1+\frac{c_{1} c_{4}}{j}\right)^{2}\left(\frac{k_{o}}{k-1}\right)^{2} \\
= & \frac{k}{(k-1)^{2}} m_{1}^{2} \prod_{j=1}^{k_{o}}\left(1+\frac{c_{1} c_{4}}{j}\right)^{2} k_{o}^{2} .
\end{aligned}
$$

Accordingly, we conclude that

$$
\lim _{k \rightarrow \infty} \operatorname{var}\left(u_{k}\right)=0 .
$$

In what follows, we characterize the mean value and the variance of $v_{k}$.

$$
\boldsymbol{E}\left[v_{k}\right]=\sqrt{k} \boldsymbol{E}\left[\sum_{i=1}^{k-1}\left(a_{i} e_{i} \prod_{j=i+1}^{k-1}\left(1-a_{j} b_{j}\right)\right)\right] .
$$

Since $e_{i}$ is independent of $b_{j}$, it follows that for any $i, j$,

$$
\boldsymbol{E}\left[v_{k}\right]=\sqrt{k} \sum_{i=1}^{k-1} \boldsymbol{E}\left[\left(a_{i} \prod_{j=i+1}^{k-1}\left(1-a_{j} b_{j}\right)\right)\right] \boldsymbol{E}\left[e_{i}\right] .
$$

Since $\boldsymbol{E}\left[e_{i}\right]=0$ for any $i \in \mathbb{N}$, we have that

$$
\boldsymbol{E}\left[v_{k}\right]=0 \text {. }
$$

To characterize the variance of $v_{k}$, observe that

$$
\begin{aligned}
& \operatorname{var}\left(v_{k}\right) \\
= & \boldsymbol{E}\left[v_{k}^{2}\right]-\boldsymbol{E}^{2}\left[v_{k}\right] \\
= & k \boldsymbol{E}\left[\left(\sum_{i=1}^{k-1} a_{i} e_{i} \prod_{j=i+1}^{k-1}\left(1-a_{j} b_{j}\right)\right)^{2}\right] .
\end{aligned}
$$

Since $e_{i}$ and $e_{j}$ are independent for $i \neq j$ and $\boldsymbol{E}\left[e_{i}\right]=0$ for any $i \in \mathbb{N}$, we have that

$$
\begin{aligned}
& \operatorname{var}\left(v_{k}\right) \\
= & k \boldsymbol{E}\left[\sum_{i=1}^{k-1} a_{i}^{2} e_{i}^{2} \prod_{j=i+1}^{k-1}\left(1-a_{j} b_{j}\right)^{2}\right] \\
= & k \boldsymbol{E}\left[\sum_{i=1}^{k_{o}-1} a_{i}^{2} e_{i}^{2} \prod_{j=i+1}^{k-1}\left(1-a_{j} b_{j}\right)^{2}\right] \\
+ & k \boldsymbol{E}\left[\sum_{i=k_{o}}^{k-1} a_{i}^{2} e_{i}^{2} \prod_{j=i+1}^{k-1}\left(1-a_{j} b_{j}\right)^{2}\right] .
\end{aligned}
$$

Combining Lemma 4.1 with (8) and (9) yields that

$$
\begin{aligned}
& k \boldsymbol{E}\left[\sum_{i=1}^{k_{o}-1} a_{i}^{2} e_{i}^{2} \prod_{j=i+1}^{k-1}\left(1-a_{j} b_{j}\right)^{2}\right] \\
\leq & k \sum_{i=1}^{k_{o}-1}\left(\frac{c_{4} c_{2}}{i}\right)^{2} \prod_{j=i+1}^{k_{o}}\left(1+\frac{c_{4} c_{1}}{j}\right)^{2} \\
& \cdot \prod_{j=k_{o}+1}^{k-1}\left(1-\frac{1}{k}\right)^{2} \\
= & \frac{k k_{o}^{2}}{(k-1)^{2}} \sum_{i=1}^{k_{o}-1}\left(\frac{c_{4} c_{2}}{i}\right)^{2} \prod_{j=i+1}^{k_{o}}\left(1+\frac{c_{4} c_{1}}{j}\right)^{2},
\end{aligned}
$$

and

$$
\begin{aligned}
& k \boldsymbol{E}\left[\sum_{i=k_{o}}^{k-1} a_{i}^{2} e_{i}^{2} \prod_{j=i+1}^{k-1}\left(1-a_{j} b_{j}\right)^{2}\right] \\
\leq & k \sum_{i=k_{o}}^{k-1}\left(\frac{c_{4} c_{2}}{i}\right)^{2} \prod_{j=i+1}^{k-1}\left(1-\frac{1}{k}\right)^{2} \\
= & \frac{k\left(k-k_{o}\right) c_{2}^{2} c_{4}^{2}}{(k-1)^{2}}
\end{aligned}
$$

It follows that

$$
\begin{aligned}
& \lim _{k \rightarrow \infty} \operatorname{var}\left(v_{k}\right) \\
\leq & \lim _{k \rightarrow \infty} \frac{k k_{o}^{2}}{(k-1)^{2}} \sum_{i=1}^{k_{o}-1}\left(\frac{c_{4} c_{2}}{i}\right)^{2} \prod_{j=i+1}^{k_{o}}\left(1+\frac{c_{4} c_{1}}{j}\right)^{2} \\
& +\lim _{k \rightarrow \infty} \frac{k\left(k-k_{o}\right) c_{2}^{2} c_{4}^{2}}{(k-1)^{2}} \\
= & c_{2}^{2} c_{4}^{2} .
\end{aligned}
$$

Therefore for $m_{k}$, we have that

$$
\begin{gathered}
\lim _{k \rightarrow \infty} \boldsymbol{E}\left[m_{k}\right]=0 \\
\lim _{k \rightarrow \infty} \operatorname{var}\left(m_{k}\right) \leq c_{2}^{2} c_{4}^{2} .
\end{gathered}
$$

Substituting $m_{k}=\sqrt{k}\left(n_{k}-n_{c}^{*}\right)$ into (11) and (12), we obtain

$$
\begin{aligned}
\lim _{k \rightarrow \infty} \boldsymbol{E}\left[n_{k}\right] & =n_{c}^{*} \\
\lim _{k \rightarrow \infty} k * \operatorname{var}\left(n_{k}-n_{c}^{*}\right) & \leq c_{3},
\end{aligned}
$$

where $c_{3}=c_{2}^{2} c_{4}^{2}$.

From Chebyshev's Inequality, we conclude that for any $\epsilon>0$,

$$
\lim _{k \rightarrow \infty} P\left(\left|n_{k}-n_{c}^{*}\right|>\epsilon\right) \leq \lim _{k \rightarrow \infty} \frac{c_{3}}{k \epsilon^{2}}=0 .
$$

Therefore $\left\{n_{k}\right\}$ converges to $n_{c}^{*}$ in probability.

\section{REFERENCES}

[1] J. Barros and S. D. Servetto. On the capacity of the reachback channel in wireless sensor networks. In Proc. IEEE Workshop on Multimedia Signal Processing (special session on "Signal Processing for Wireless Networks"), pages 408 - 411, St. Thomas, Virgin Islands, USA, December 2002.

[2] C. Budianu, S. Ben-David, and L. Tong. Estimation of number of operating sensors in large-scale sensor networks with mobile access. IEEE Transactions on Signal Processing, to appear, 2005. 
[3] E. Duarte-Melo and M. Liu. Energy efficiency in many-to-one communications in wireless networks. In Proc. IEEE 45th Midwest Symposium on Circuits and Systems, Oklahoma State University, Tulsa, Oklahoma, August 2002.

[4] E. Duarte-Melo, M. Liu, and A. Misra. An efficient and robust computational framework for studying lifetime and information capacity in sensor networks. ACM Mobile Networks and Applications (MONET) special issue on Energy Constraints and Lifetime Performance in Wireless Sensor Networks, 2004.

[5] M. Gastpar and M. Vetterli. Power, spatio-temporal bandwidth, and distortion in large sensor networks. IEEE Journal on Selected Areas in Communications, to appear, 2005.

[6] Isaacson and Madsen. Markov Chains theory and applications. John Wiley \& Sons, Inc., first edition, 1976.

[7] Ranjit Iyer and Leonard Kleinrock. QoS control for sensor networks. In Proc. IEEE International Conference on Communications, pages 517-521, May 2003.

[8] A. Leshem and L. Tong. Estimating sensor population via probabilistic sequential polling. IEEE Signal Processing Letters, 12(5):395-398, May 2005.

[9] Daniel Marco, E. Duarte-Melo, M. Liu, and David L. Neuhoff. On the many-to-one transport capacity of a dense wireless sensor network and the compressibility of its data. In Proc. International Workshop on Information Processing in Sensor Networks (IPSN), Palo Alto Research Center (PARC), Palo Alto, California, US, April 2003.

[10] Y. Rachlin, R. Negi, and P. Khosla. Sensing capacity for discrete sensor network applications. In Proc. the Fourth International Symposium on Information Processing in Sensor Networks (IPSN), UCLA, Los Angeles, California, USA, April 2005.

[11] Z. Yang and L. Tong. Capacity of cooperative sensor networks with sensor errors. In Proc. IEEE International Conference on Communications (ICC), Seoul, Korea, May 2005.

[12] C. Yuen and P. Marbach. Price-based rate control in random access networks. IEEE/ACM Transactions on Networking, to appear.

[13] Q. Zhao and L. Tong. Energy-efficient information retrieval for correlated source reconstruction in sensor networks. submitted to IEEE Transactions on Wireless Communications, 2004. 